\title{
Intra-night optical variability characteristics of different classes of narrow line Seyfert 1 galaxies
}

\author{
Kshama S. K. ${ }^{1},{ }^{\star}$ Vaidehi S. Paliya ${ }^{2}$, C. S. Stalin ${ }^{1}$ \\ ${ }^{1}$ Indian Institute of Astrophysics, Block II, Koramangala, Bangalore, 560034, India \\ ${ }^{2}$ Department of Physics and Astronomy, Clemson University, 118 Kinard Laboratory, Clemson, S.C. 29634-0978, USA
}

Accepted XXX. Received YYY; in original form ZZZ

\begin{abstract}
In a first systematic effort to characterize the intra-night optical variability (INOV) of different classes of narrow line Seyfert 1 (NLSy1) galaxies, we have carried out observations on a sample of radio-loud (RL) and radio-quiet (RQ) NLSy1 galaxies. The RL-NLSy1 galaxies are further divided into $\gamma$-ray loud (GL) and $\gamma$-ray quiet (GQ) NLSy1 galaxies. Our sample consists of four sets, each set consisting of a RQ-NLSy1, a GQ-NLSy1 and a GL-NLSy1 galaxy, closely matched in redshift and optical luminosity. Our observations on both RQ and GQ-NLSy1 galaxies consist of a total of 19 nights, whereas the data for GL-NLSy1 galaxies (18 nights) were taken from literature published earlier by us. This enabled us to do a comparison of the duty cycle (DC) of different classes of NLSy1 galaxies. Using power-enhanced F-test, with a variability threshold of 1\%, we find DCs of about 55\%, 39\% and $0 \%$ for GL, GQ and RQ-NLSy1 galaxies respectively. The high DC and large amplitude of INOV (24.0 \pm $13.7 \%$ ) shown by GL-NLSy1 galaxies relative to the other two classes might be due to their inner aligned relativistic jets having large bulk Lorentz factors. The null DC of RQ-NLSy1 galaxies could mean the presence of low power and/or largely misaligned jets in them. However, dividing RL-NLSy1 galaxies into low and high optical polarization sources, we find that sources with large polarization show somewhat higher DCs (69\%) and amplitudes (29\%) compared to those with low polarization. This points to a possible link between INOV and optical polarization.
\end{abstract}

Key words: surveys - galaxies:active - galaxies:Seyferts- galaxies: jets - galaxies: photometry.

\section{INTRODUCTION}

One of the defining characteristics of active galactic nuclei (AGN) is that they show intensity variations over the complete accessible electromagnetic spectrum from low energy radio waves to high energy $\gamma$-rays (Wagner \& Witzel 1995). Such variations are known to occur on time scales ranging from minutes to hours to days (Miller et al. 1989; Heidt \& Wagner 1996; Abdo et al. 2010). Variability, particularly those observed at short time scales of the order of minutes, can be an efficient probe of AGN structure as well as the physical processes happening close to their central engine not accessible to conventional imaging techniques. Among AGN, blazars (that comprise both flat spectrum radio quasars (FSRQs) and BL Lacertae (BL Lac) objects) whose jets are pointed close to our line of sight (Urry \& Padovani 1995) are known to show violent (large amplitude and short time scale) variations. Such short time scale variations in blazars are known across the electromag-

^ E-mail: kshama.kurian@iiap.res.in netic spectrum including $\gamma$-rays (Ackermann et al. 2016) and Xrays (Paliya et al. 2015) as well as optical (Sagar et al. 2004).

Apart from blazars, other classes of AGN too are known to exhibit rapid intensity fluctuations in the optical, hereafter termed as intra-night optical variability (INOV). Example includes the reports of INOV in radio-quiet quasars (Gopal-Krishna et al. 2003), lobe dominated quasars (Stalin et al. 2004b) and the weak line quasars (Gopal-Krishna et al. 2013). Thus, it is now convincingly established that different classes of quasars show INOV and among them blazars show large amplitude and high duty cycle (DC) of INOV compared to radio-quiet quasars that show low amplitude and low DC of INOV (Stalin et al. 2004a). The detection of INOV in blazars is usually explained by invoking shocks propagating down their relativistic jets (Marscher \& Gear 1985). Alternative models of intrinsic flux variations in blazars include instabilities or fluctuations in relativistic jet flows (Hughes et al. 1992; Marscher et al. 1992). The clear existence of INOV (albeit with low amplitude and low DC) in radio-quiet quasars, had led to the idea of the presence of micro-arcsec scale optical synchrotron jets in them (Gopal-Krishna et al. 2003). Hints for the presence of weak ra- 
dio jets in radio-quiet quasars are known from deep VLA observations (Blundell \& Rawlings 2001; Miller et al. 1993) as well as from the presence of hard X-ray tail in some radio-quiet quasars (George et al. 2000).

Though the presence of INOV in luminous quasars is well established (Miller et al. 1989; Sagar et al. 2004), their presence in the less luminous counterparts, the Seyfert galaxies is not very clear. Though Seyfert galaxies are known since the discovery of AGN, a new class of Seyfert galaxies called Narrowline Seyfert 1 galaxies (NLSy1) were discovered about three decades ago by Osterbrock \& Pogge (1985). In the optical spectrum these source have emission lines from the broad line region narrower than Seyfert 1 galaxies but broader than Seyfert 2 galaxies (Rodríguez-Ardila et al. 2000) with FWHM $\left(H_{\beta}\right)$ less than 2000 $\mathrm{km} \mathrm{sec}{ }^{-1}$. They have weak OIII $\left(O I I I / H_{\beta}<3\right)$ and strong Fe II lines. In addition to the narrow permitted lines from the broad line region (attributed to low black hole mass), NLSy1 galaxies have excess soft X-ray emission and steeper spectra in X-rays when compared to broad line Seyfert 1 galaxies. They also show high accretion rates, close to the Eddington limit (Boroson 2002). In the radio regime, NLSy1 galaxies are found to be mostly radioquiet. However, Komossa et al. (2006) found that about $7 \%$ of the NLSy1 galaxies are radio-loud having the radio loudness parameter $R>10$ ( $\mathrm{R}$ is defined as the ratio of the radio flux density at 5 $\mathrm{GHz}$ to the optical flux density at $440 \mathrm{~nm}$ (Kellermann et al. 1989) and among them about $2 \%-3 \%$ are very radio-loud $(\mathrm{R}>100)$. This is much smaller than the radio-loud fraction of $15 \%$ known in the quasar category of AGN (Urry \& Padovani 1995). The small fraction can be due to 'selection effects' as the sample was selected based on $F W H M(H \beta)<2000 \mathrm{~km} \mathrm{~s}^{-1}$ irrespective of the luminosity of the source (Komossa et al. 2006). While a majority of radioloud NLSy1 (RL-NLSy1) galaxies are known to have a flat radio spectrum, some of them are found to be compact steep spectrum sources. There are also a few reports of rapid optical flux variations in NLSy1 galaxies (Miller et al. 2000; Klimek et al. 2004).

After the launch of the Fermi Gamma-ray Space Telescope (hereafter Fermi), in the year 2008, the Large Area Telescope (LAT) aboard Fermi detected for the first time $\gamma$-ray emission from the RL-NLSy1 galaxy, PMN J0948+0022 (Abdo et al. 2009a). This gave proof of the presence of relativistic jet in PMN J0948+0022. Soon after the detection in $\gamma$-rays, INOV with an amplitude of variability of 0.5 mag in few hours was reported by Liu et al. (2010) in PMN J0948+0022. It was also found to be variable by Maune et al. (2011). Rapid variations have also been reported in the infra-red bands by Jiang et al. (2012). Subsequent to the report on the detection of $\gamma$-ray emission in PMN J0948+0022, few more RL-NLSy1 galaxies were also reported to be emitters of $\gamma$-rays by Fermi. During the start of this observational program five NLSy1 galaxies were known to be $\gamma$-ray emitters (Foschini et al. 2011, 2015) with high significance having test statistics (TS) $>100$ and the $\gamma$-ray flux in the $0.1-100 \mathrm{GeV}$ band larger than $5 \times 10^{-8} \mathrm{ph} \mathrm{cm}^{-2} \mathrm{~s}^{-1}$. A $10 \sigma$ detection corresponds to a TS value of 100 (Mattox et al. 1996). Since then more $\gamma$-ray emitting RL-NLSy1 galaxies have been discovered, such as 1FHL J1410.4+7408 (Marchesini et al. 2016), FBQS J1644+2619 (D'Ammando et al. 2015) and SDSS J122222.55+041315.7 (Yao et al. 2015). The RL-NLSy1 galaxies which emit $\gamma$-rays are hereafter referred to as $\gamma$-ray loud NLSy1 (GL-NLSy1) galaxies. Majority of the GL-NLSy1 galaxies have been studied for INOV and from the observations as of now available in the literature, it is clear that their INOV properties are similar to blazars (Liu et al. 2010; Paliya et al. 2013a, 2014, 2016). Strong and variable optical polarization are also known in three
GL-NLSy1 galaxies (Maune et al. 2014; Itoh et al. 2013, 2014). The broad band spectral energy distribution of GL-NLSy1 galaxies resemble FSRQs (Abdo et al. 2009b; Paliya et al. 2013b, 2014).

High quality observations exist on the INOV properties of radio-loud and radio-quiet quasars. These studies present ample indirect evidence of the presence of relativistic miniature (parsec scale) jets in radio-quiet quasars. If this observational evidence is considered to be present in the less luminous NLSy1 galaxies, it is natural to expect both RL and radio-quiet NLSy1 (RQ-NLSy1) galaxies to show INOV, though the amplitude and the prevalence of INOV will be different among them. Incidentally, the high DC and amplitude of INOV detected in GL-NLSy1 galaxies reinforces their similarity to blazars and also argues for the detectability of INOV in RQ-NLSy1 galaxies. However, as of now, INOV studies of other classes of NLSy1 galaxies are limited to few nights of observations (Miller et al. 2000; Klimek et al. 2004). There is thus a need for systematic INOV observations of different classes of NLSy1 galaxies (a) to establish the presence of INOV in RQ-NLSy1 galaxies and (b) to compare the INOV of RQ-NLSy1 galaxies with their radio-loud counterparts. To achieve the above stated objectives we have carried out a programme of systematic INOV observations of a sample of NLSy1 galaxies. We present here the results of that programme.

\section{OBSERVATIONS AND DATA REDUCTION}

\subsection{Sample set}

Our sample consists of radio-loud and radio-quiet NLSy1 galaxies. The RL-NLSy1 galaxies are further divided into $\gamma$-ray loud and $\gamma$-ray quiet (GQ) NLSy1 galaxies based on the detection of $\gamma$-rays by Fermi. There are four sets of NLSy1 galaxies. This was driven by the number of GL-NLSy1 galaxies known as well as their observability using the $2 \mathrm{~m}$ Himalayan Chandra Telescope (HCT) at Hanle, during the time this monitoring program was initiated. Each set consists of a GL-NLSy1, a GQ-NLSy1 and a RQ-NLSy1 galaxy. To avoid selection biases due to differences in luminosity and redshift, objects were selected such that all sources in a particular set have similar optical brightness and redshift. The four GLNLSy1 galaxies are taken from the papers already published by us in Paliya et al. (2013a, 2016). The remaining four RQ NLSy1 galaxies and GQ-NLSy1 galaxies each were taken from the catalogue of NLSy1 galaxies by Zhou et al. (2006). Our entire sample thus consists of a total of 12 sources spanning the redshift range $0.06<z<0.66$.

The RQ-NLSy1 galaxies in each set are selected such that they are truly radio-quiet and not-detected at $1.4 \mathrm{GHz}$ at $1 \mathrm{mJy}$ level in the FIRST ${ }^{1}$ survey. However, GQ-NLSy1 galaxies are those that have a significant detection in FIRST. For the GQ and GL-NLSy1 galaxies we estimated the radio-loudness $\mathrm{R}$ parameter using the formula given by Ivezić et al. (2002)

$\log R=\log \left(\frac{F_{r}}{F_{\text {opt }}}\right)=0.4(m-t)$

where $m$ is the SDSS r-band magnitude and $t$ is the radio AB magnitude defined as

$t=-2.5 \log \left(\frac{F_{\text {int }}}{3631 J y}\right)$

\footnotetext{
1 Faint Images of the Radio Sky at Twenty cm: http://sundog.stsci.edu
} 
here, $F_{\text {int }}$ is the integrated radio flux and $3631 \mathrm{Jy}$ is the zero point. It is derived using the following relation by Oke (1974)

$m_{A B}=-2.5 \log \left(f_{v}\right)-48.60$

here, $f_{v}$ is flux density in erg $\mathrm{cm}^{-2} \mathrm{~s}^{-1} \mathrm{~Hz}^{-1}$. The properties of the objects observed for INOV are given in Table 1.

\subsection{Observations}

The observations of the RQ and GQ-NLSy1 galaxies in our sample were carried out over a period of 2 years from 2012 August to 2014 September, using the $2 \mathrm{~m} \mathrm{HCT}$ operated by the Indian Institute of Astrophysics, Bangalore. It is a Ritchey-Chretien system with f/9 Cassegrain focus. The CCD detector has a gain of $1.22 \mathrm{e}^{-}$and readout noise of $4.8 \mathrm{e}^{-} / A D U$. The field of view is $10^{\prime} \times 10^{\prime}$. Each pixel of the CCD corresponds to $0.3^{\prime \prime} \times 0.3^{\prime \prime}$ of the sky ${ }^{2}$. The objects were observed in Bessel $\mathrm{R}$ band as it gives the best $\mathrm{S} / \mathrm{N}$ ratio. The typical $\mathrm{S} / \mathrm{N}$ ratio in our observations is around 150 and the time resolution is of the order of 300 seconds for most of the objects. According to Carini (1990), the probability of detecting INOV in a source is enhanced if it is observed continuously for a duration of about 3-4 hours on any given night. Hence, we tried to monitor each source for greater than 3 hours continuously whenever possible. Also, each source was suitably pointed to have two or more comparison stars within a magnitude of the target NLSy1 galaxies. The log of observations is given in Table 2 .

\subsection{Data Reduction}

Preliminary processing (bias subtraction and flat fielding) of the observations as well as photometry were done using the IRAF software ${ }^{3}$. Instrumental magnitudes of the target NLSy1 galaxies and the comparison stars were determined by aperture photometry using the phot task in APPHOT package in IRAF. At least three comparison stars (usually more) with similar brightness to the NLSy1 galaxies were considered in each frame. A crucial parameter to the phot task is the radius of the aperture for photometry and this determines the $\mathrm{S} / \mathrm{N}$ ratio of any object in the observed frames. According to Howell (1989) the $\mathrm{S} / \mathrm{N}$ ratio of a source in a CCD frame is maximum when the radius of the photometric aperture is approximately equal to the full width at half maxima (FWHM) of the point spread function (PSF) of the objects and decreases for both larger and smaller apertures. Hence, to select the optimum aperture that maximizes the $\mathrm{S} / \mathrm{N}$, for each night, a range of aperture radii was considered and the aperture that minimizes the standard deviation of the differential light curve (DLC) of the steadiest pair of comparison stars was taken as the optimum aperture for photometry of the target NLSy1 galaxies for that night (Stalin et al. 2004a). The standard deviation of the DLC of the steadiest pair of comparison stars thus constructed will provide the actual error in the photometry, as the photometric errors reported by IRAF are an underestimate (Goyal et al. 2013). The positions and the apparent B and R magnitudes (the uncertainties of which can be up to $0.25 \mathrm{mag}$ ) taken from the USNO-B2 ${ }^{4}$ catalogue, of the comparison stars for each of the sources, are mentioned in Table 3.

2 http://www.iiap.res.in/centers/iao

3 IRAF is distributed by the National Optical Astronomy Observatories, which is operated by the Association of Universities for Research in Astronomy Inc. under cooperative agreement with the National Science Foundation

${ }^{4}$ http://www.nofs.navy.mil/data/fchpix/

\section{ANALYSIS}

Using the instrumental magnitudes obtained from photometry, DLCs of the NLSy1 galaxies were generated relative to a minimum of three comparison stars, using an optimum aperture that is close to the median FWHM on that night. In most of the NLSy1 galaxies in our sample, the host galaxy has negligible effects on the photometry of the objects (Cellone et al. 2000), as the central AGN dominates. However, for the NLSy1 galaxies in Set 1 (lowest redshift bin), it is likely that the underlying host galaxy may affect the flux variations of the NLSy1 galaxy owing to varying seeing conditions. As noted by Carini et al. (1991), for sources with underlying host galaxy components, any spurious variations introduced by fluctuations in atmospheric seeing are typically smaller than the observational uncertainties. Even so, for unambiguous detection of INOV and to rule out any spurious variations contributed by the hosts of the NLSy1 galaxies, we have checked for any close correspondence between the seeing variations in any particular night and the DLCs of the NLSy1 galaxies relative to the comparison stars. We find that the host galaxies have negligible effect on the photometry reported here. We define INOV as flux variations greater than or of the order of $1 \%$ which corresponds to $95 \%$ confidence ( $2 \sigma$ level). This is driven by the error in our photometry which is typically around 0.005 magnitudes. To check for the presence or absence of INOV in a source, we have used the power-enhanced F test (de Diego 2014).

\subsection{Power-enhanced F-test}

This test is proposed to be free from the difficulties associated with the widely used criteria to test INOV, such as the C and F statistics (de Diego 2014) and has found increased usage in recent studies of INOV in AGN (Gaur et al. 2015; Polednikova et al. 2016). It consists of transforming the comparison star's DLCs to have the same photometric noise, as if their magnitudes exactly matched the mean magnitude of the AGN under study. The enhanced F, statistical criterion is defined as

$F_{\text {enh }}=\frac{s_{q s o}^{2}}{s_{c}^{2}}$

Here, $s_{q s o}^{2}$ is the variance of the NLSy1 galaxy-reference star DLC and $s_{c}^{2}$ is the stacked variance (de Diego 2014) of the comparison star-reference star DLCs given as

$s_{c}^{2}=\frac{1}{\left(\sum_{j=1}^{k} N_{j}-k\right)} \sum_{j=1}^{k} \sum_{i=1}^{N_{j}} s_{j, i}^{2}$

where $N_{j}$ is the number of observations of the $j^{\text {th }}$ star, $k$ is the total number of comparison stars. $s_{j, i}^{2}$ is the scaled square deviation, defined as

$s_{j, i}^{2}=\omega_{j}\left(m_{j, i}-\bar{m}_{j}\right)^{2}$

where, $\omega_{j}$ is the scaling factor, $m_{j, i}$ 's are the differential instrumental magnitudes and $\bar{m}_{j}$ is the mean differential magnitude of the reference star and the $j^{\text {th }}$ comparison star. Following Joshi et al. (2011), we have taken $\omega_{j}$ as the ratio of the averaged square error of the differential instrumental magnitudes in the NLSy1 galaxy reference star DLC $\left(\left\langle\sigma_{q}^{2}\right\rangle\right)$ to the averaged square error of the differential instrumental magnitudes in the comparison star - reference star DLC $\left(<\sigma_{s_{j}}^{2}>\right)$. For the $j^{\text {th }}$ star DLC

$\omega_{j}=\frac{<\sigma_{q}^{2}>}{<\sigma_{s_{j}}^{2}>}$ 


\section{$4 \quad$ Kshama et al.}

Table 1. Details of the NLSy1 galaxies that are monitored in this program. Column information are as follows: (1)IAU Name (2) Other name (3)right ascension (4) declination (5) R-band magnitude (6) redshift (7) type (8) integrated flux from FIRST (here ND refers to 'Not detected' in FIRST survey) (9) radio loudness parameter and (10) optical polarization

\begin{tabular}{|c|c|c|c|c|c|c|c|c|c|}
\hline $\begin{array}{l}\text { Name } \\
\text { (1) }\end{array}$ & $\begin{array}{l}\text { Other Name } \\
\text { (2) }\end{array}$ & $\begin{array}{l}\alpha_{2000} \\
(3)\end{array}$ & $\begin{array}{l}\delta_{2000} \\
(4)\end{array}$ & $\begin{array}{l}\mathrm{R} \\
\text { (mag) } \\
(5)\end{array}$ & $\begin{array}{l}z \\
\text { (6) }\end{array}$ & $\begin{array}{l}\text { Type } \\
\text { (7) }\end{array}$ & $\begin{array}{l}F_{\text {int }} \\
(\mathrm{mJy}) \\
(8)\end{array}$ & $\begin{array}{l}\log R \\
(9)\end{array}$ & $\begin{array}{l}\mathrm{P}_{o p t} \\
(\%) \\
(10)\end{array}$ \\
\hline \multicolumn{10}{|l|}{ Set-1 } \\
\hline $\mathrm{J} 0324+3410$ & 1H $0323+342$ & $03: 24: 41.10$ & $+34: 10: 46.0$ & 15.7 & 0.061 & GL-NLSy1 & $559^{*}$ & 2.468 & $3^{a}$ \\
\hline $\mathrm{J} 2219+1207$ & II Zw 177 & $22: 19: 18.53$ & $+12: 07: 52.2$ & 16.4 & 0.080 & GQ-NLSy1 & 1.51 & 0.196 & - \\
\hline J0351-0526 & 2MASX J03510759-0526370 & 03:51:07.61 & $-05: 26: 37.1$ & 16.1 & 0.069 & RQ-NLSy1 & ND & - & - \\
\hline \multicolumn{10}{|l|}{ Set-2 } \\
\hline $\mathrm{J} 0849+5108$ & SBS $0846+513$ & $08: 49: 58.00$ & $+51: 08: 29.0$ & 18.3 & 0.586 & GL-NLSy1 & 350.53 & 3.3 & $10^{b}$ \\
\hline $\mathrm{J} 1613+5247$ & SDSS J161301.86+524749.5 & $16: 13: 01.88$ & $+52: 47: 49.5$ & 18.7 & 0.530 & GQ-NLSy1 & 1.35 & 1.04 & - \\
\hline $\mathrm{J} 2123+0102$ & SDSS J212309.51+010213.0 & $21: 23: 09.51$ & $+01: 02: 13.1$ & 19.2 & 0.590 & RQ-NLSy1 & ND & - & - \\
\hline \multicolumn{10}{|l|}{ Set-3 } \\
\hline $\mathrm{J} 0948+0022$ & PMN J0948+0022 & $09: 48: 57.30$ & $+00: 22: 26.0$ & 18.4 & 0.585 & GL-NLSy1 & 111.46 & 2.892 & $36^{c}$ \\
\hline J2339-0912 & FBQS J2339-0912 & $23: 39: 03.82$ & $-09: 12: 21.2$ & 19.3 & 0.660 & GQ-NLSy1 & 4.39 & 1.788 & - \\
\hline $\mathrm{J} 1326+0334$ & SDSS J132654.80+033456.3 & $13: 26: 54.81$ & $+03: 34: 56.3$ & 18.7 & 0.588 & RQ-NLSy1 & ND & - & - \\
\hline \multicolumn{10}{|l|}{ Set-4 } \\
\hline $\mathrm{J} 1505+0326$ & PKS $1502+036$ & $15: 05: 06.50$ & $+03: 26: 31.0$ & 18.2 & 0.409 & GL-NLSy1 & 380.49 & 3.312 & - \\
\hline $\mathrm{J} 1256+3852$ & FBQS J1256+3852 & $12: 56: 02.10$ & $+38: 52: 31.0$ & 17.4 & 0.419 & GQ-NLSy1 & 2.1 & 0.744 & - \\
\hline J0037-0933 & SDSS J003753.72-093331.8 & $00: 37: 53.73$ & $-09: 33: 31.8$ & 18.9 & 0.399 & RQ-NLSy1 & ND & - & - \\
\hline
\end{tabular}

* value taken from (Véron-Cetty \& Véron 2010), ${ }^{a}$ (Itoh et al. 2014), ${ }^{b}$ (Maune et al. 2014), ${ }^{c}$ (Itoh et al. 2013)

It is now known that the photometric errors returned by IRAF are underestimated (Goyal et al. 2013; Stalin et al. 2004a) by factors of about 1.5. But as $\omega_{j}$ is the ratio of the errors, this factor gets cancelled out. Thus by stacking the variances of the comparison stars, the degrees of freedom of the denominator of the F-distribution given in Equation 4 increases, which in turn increases the power of the test (de Diego et al. 2015). In this work, for most of the nights we have three stars from which the star with magnitude as close as possible or brighter than the NLSy1 galaxy in the field is taken as the reference star. Hence we are left with two stars as the comparison stars. The quasar and comparison stars have the same number of observations and hence the number of degrees of freedom of the numerator and denominator in Equation 4 are $v_{1}=N-1$ and $v_{2}=k(N-1)$ respectively. The $F_{\text {enh }}$ value is then estimated and compared with the critical value of $\mathrm{F}\left(F_{c}\right)$ for $\alpha=0.05$ which corresponds to $95 \%$ confidence level. A source is considered variable if $F_{e n h}$ is greater than or equal to the calculated $F_{c}$. The estimated values of $F_{\text {enh }}$ are given in Table 4 .

\subsection{Amplitude of Variability}

For objects that are found to satisfy the adopted statistical criterion, we calculated the variability amplitude (Amp) following Heidt \& Wagner (1996). Amp is defined as

$A m p=\sqrt{\left(A_{\max }-A_{\min }\right)^{2}-2 \sigma^{2}}$

here, $A_{\max }$ is the maximum in the NLSy1 galaxy - reference star DLC, $A_{\min }$ is the minimum in the NLSy1 galaxy - reference star DLC and $\sigma$ is the standard deviation of the steadiest comparison star-reference star DLC. The calculated amplitude of variability for the variable NLSy1 galaxies are shown in Table 4. Table 5 shows the mean variability amplitude and the associated error in amplitude for the different types of NLSy1 galaxies.

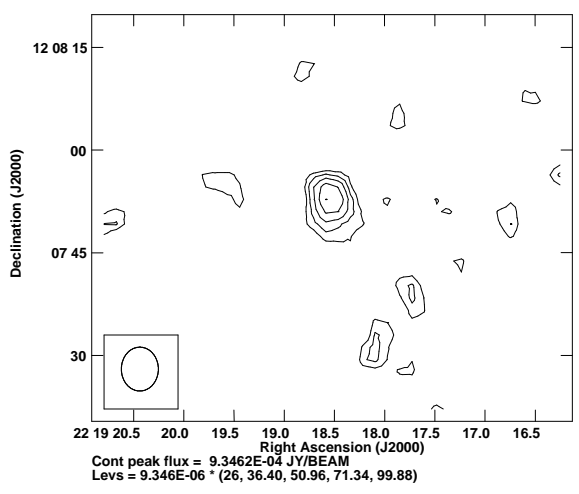

Figure 1. FIRST image of the GQ-NLSy1 galaxy, J2219+1207.

\subsection{Duty Cycle}

AGN do not show variability at all times they are monitored. Therefore, duty cycle (DC) of variability of each class of NLSy1 galaxy is estimated by the ratio of the time over which each object in a given class is found to be variable to the total observing time spent on monitoring the objects in a class. As per the definition given by Romero et al. (1999), the DC is calculated as

$D C=100 \frac{\sum_{n}^{i=1} N_{i}\left(1 / \Delta t_{i}\right)}{\sum_{n}^{i=1}\left(1 / \Delta t_{i}\right)} \%$

where $\Delta t_{i}=\Delta t_{i, o b s}(1+z)^{-1}$ is the duration of monitoring session of a source on the $\mathrm{i}^{\text {th }}$ night, corrected for its cosmological redshift $z$. $N_{i}$ is set to 1 if INOV is detected, else it is set to 0 . The results of DC for different classes of NLSy1 galaxies are given in Table 5. 

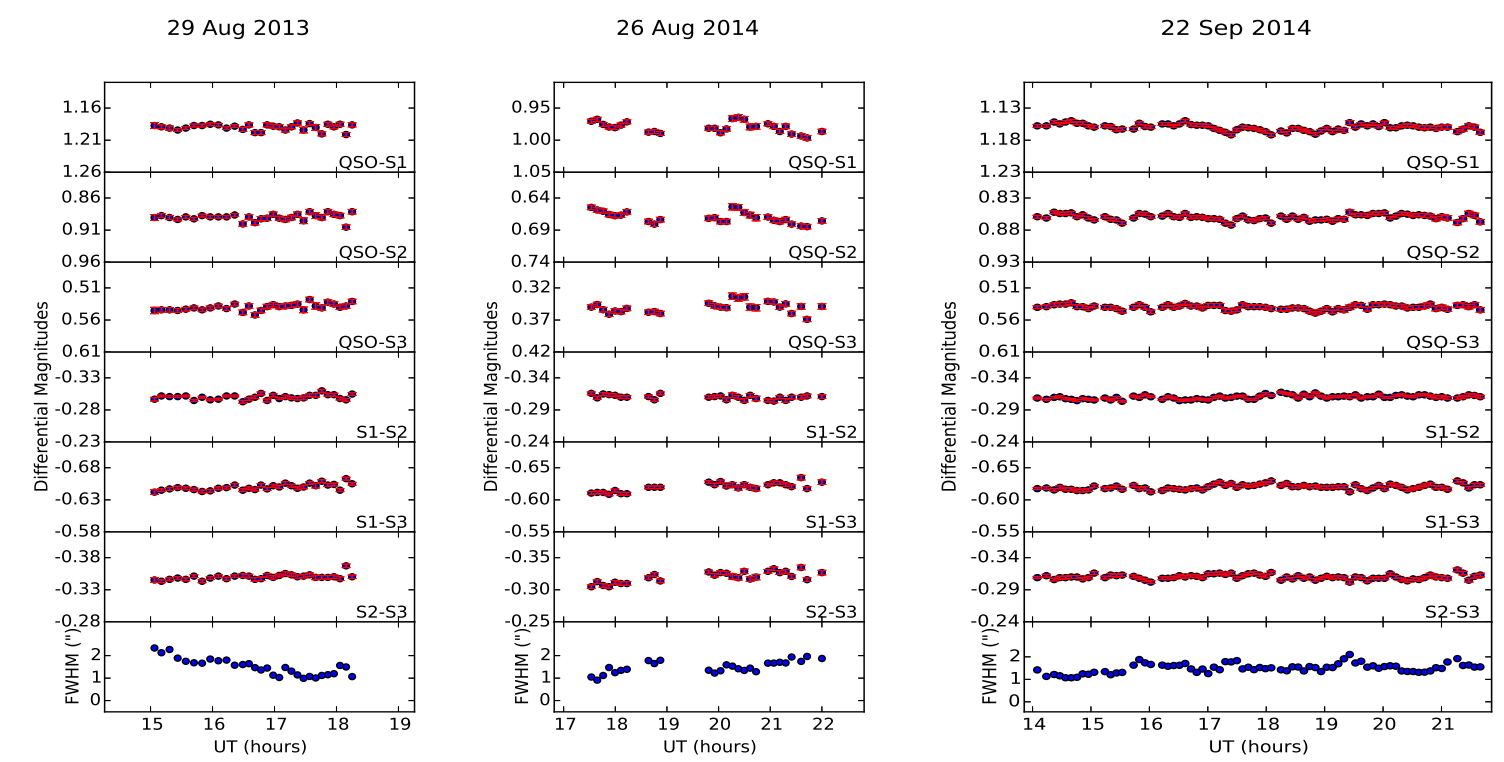

Figure 2. DLCs of the GQ-NLSy1 galaxy, J2219+1207.

Table 2. Log of observations. Columns information are as follows: (1) IAU name (2) date of observation (3) duration of observation and (4) number of data points

\begin{tabular}{llll}
\hline Name & Date & $\begin{array}{c}\Delta \mathrm{T} \\
\text { (hours) }\end{array}$ & $\mathrm{N}$ \\
$(1)$ & $(2)$ & $(3)$ & $(4)$ \\
\hline $\mathrm{J} 2219+1207$ & $29 / 08 / 2013$ & 3.2 & 30 \\
& $26 / 08 / 2014$ & 4.5 & 27 \\
& $22 / 09 / 2014$ & 7.6 & 73 \\
\hline $\mathrm{J} 0351-0526$ & $01 / 02 / 2014$ & 2.9 & 11 \\
& $02 / 02 / 2014$ & 2.7 & 20 \\
\hline $\mathrm{J} 1613+5247$ & $03 / 07 / 2013$ & 4.7 & 13 \\
& $12 / 07 / 2013$ & 6.1 & 25 \\
& $31 / 03 / 2014$ & 6.3 & 26 \\
\hline $\mathrm{J} 2123+0102$ & $11 / 08 / 2012$ & 5.3 & 28 \\
& $03 / 06 / 2013$ & 2.8 & 13 \\
\hline $\mathrm{J} 2339-0912$ & $05 / 10 / 2013$ & 6.4 & 24 \\
\hline $\mathrm{J} 1326+0334$ & $30 / 03 / 2014$ & 6.6 & 27 \\
\hline $\mathrm{J} 1256+3852$ & $10 / 04 / 2013$ & 3.2 & 23 \\
& $05 / 01 / 2014$ & 3.4 & 10 \\
& $01 / 02 / 2014$ & 2.5 & 9 \\
& $02 / 02 / 2014$ & 1.6 & 9 \\
& $01 / 05 / 2014$ & 2.8 & 20 \\
\hline J0037-0933 & $16 / 10 / 2012$ & 5.6 & 12 \\
& $06 / 10 / 2013$ & 6.0 & 21 \\
\hline & & &
\end{tabular}

\section{NOTES ON INDIVIDUAL OBJECTS MONITORED IN THIS WORK}

Set 1 - J2219+1207: This is a compact radio-loud source having an integrated flux density of $1.51 \mathrm{mJy}$ at $1.4 \mathrm{GHz}$. The radio con-
Table 3. Properties of the comparison stars used in differential photometry. Column information are as follows: (1) IAU name (2) Star identification(S) (3) right ascension (4) declination (5) B and (6) R magnitudes

\begin{tabular}{|c|c|c|c|c|c|}
\hline $\begin{array}{l}\text { IAU Name } \\
\text { (1) }\end{array}$ & (2) & $\begin{array}{c}\alpha_{2000} \\
\text { (3) }\end{array}$ & $\begin{array}{c}\delta_{2000} \\
(4)\end{array}$ & $\begin{array}{c}\text { B } \\
(\mathrm{mag}) \\
(5)\end{array}$ & $\begin{array}{c}\mathrm{R} \\
(\mathrm{mag}) \\
(6)\end{array}$ \\
\hline \multirow[t]{3}{*}{$\mathrm{J} 2219+1207$} & S1 & $22: 19: 26.09$ & $+12: 05: 17.10$ & 16.27 & 14.17 \\
\hline & $\mathrm{S} 2$ & $22: 19: 30.62$ & $+12: 04: 34.68$ & 16.10 & 14.46 \\
\hline & $\mathrm{S} 3$ & $22: 19: 21.36$ & $+12: 10: 07.77$ & 16.62 & 14.76 \\
\hline \multirow{3}{*}{ J0351-0526 } & $\mathrm{S} 1$ & 03:51:00.82 & $-05: 26: 04.03$ & 16.15 & 14.21 \\
\hline & $\mathrm{S} 2$ & 03:51:13.20 & $-05: 23: 19.85$ & 16.13 & 13.93 \\
\hline & S3 & 03:50:57.94 & $-05: 27: 45.46$ & 17.14 & 14.96 \\
\hline \multirow[t]{3}{*}{$\mathrm{J} 1613+5247$} & $\mathrm{~S} 1$ & $16: 12: 42.85$ & $+52: 46: 30.54$ & 18.10 & 17.8 \\
\hline & $\mathrm{S} 2$ & $16: 13: 05.83$ & $+52: 44: 33.85$ & 20.88 & 18.87 \\
\hline & S3 & $16: 13: 00.55$ & $+52: 45: 19.09$ & 20.89 & 19.28 \\
\hline \multirow[t]{3}{*}{$\mathrm{J} 2123+0102$} & $\mathrm{~S} 1$ & $21: 23: 03.32$ & $+01: 02: 56.14$ & 18.56 & 16.59 \\
\hline & $\mathrm{S} 2$ & $21: 23: 19.18$ & $+01: 04: 14.39$ & 17.88 & 16.15 \\
\hline & $\mathrm{S} 3$ & $21: 23: 13.89$ & $+01: 07: 52.62$ & 20.76 & 18.53 \\
\hline \multirow[t]{3}{*}{ J2339-0912 } & $\mathrm{S} 1$ & $23: 38: 52.98$ & $-09: 13: 19.34$ & 20.22 & 17.84 \\
\hline & $\mathrm{S} 2$ & 23:39:02.42 & $-09: 11: 45.71$ & 18.57 & 17.88 \\
\hline & $\mathrm{S} 3$ & 23:39:09.57 & -09:08:33.28 & 17.52 & 16.94 \\
\hline \multirow[t]{3}{*}{$\mathrm{J} 1326+0334$} & S1 & $13: 26: 45.36$ & $+03: 33: 30.48$ & 20.64 & 18.78 \\
\hline & $\mathrm{S} 2$ & $13: 26: 53.83$ & $+03: 34: 34.47$ & 19.57 & 18.08 \\
\hline & S3 & $13: 26: 52.20$ & $+03: 33: 04.39$ & 18.26 & 17.05 \\
\hline \multirow[t]{3}{*}{$\mathrm{J} 1256+3852$} & $\mathrm{~S} 1$ & $12: 55: 46.11$ & $+38: 49: 00.05$ & 17.25 & 16.86 \\
\hline & $\mathrm{S} 2$ & $12: 56: 14.05$ & $+38: 48: 58.61$ & 16.80 & 15.94 \\
\hline & $\mathrm{S} 3$ & $12: 56: 16.92$ & $+38: 48: 20.20$ & 18.44 & 16.77 \\
\hline \multirow[t]{3}{*}{ J0037-0933 } & $\mathrm{S} 1$ & $00: 37: 40.28$ & $-09: 32: 40.10$ & 18.84 & 17.89 \\
\hline & $\mathrm{S} 2$ & 00:37:44.14 & $-09: 33: 25.37$ & 19.13 & 17.65 \\
\hline & S3 & $00: 37: 50.49$ & $-09: 30: 50.38$ & 19.47 & 18.83 \\
\hline
\end{tabular}


Table 4. Results of variability analysis. NV: non-variable and V: variable. Column information are as follows (1) IAU name (2) type (3) date of observation (4) reference star (5) comparison stars (6) degrees of freedom of numerator $\left(v_{1}\right)$ and denominator $\left(v_{2}\right)(7)$ enhanced F test value $(8)$ critical F value for $\alpha=0.05$ (9) enhanced F statistics variability status (10) amplitude of variability

\begin{tabular}{|c|c|c|c|c|c|c|c|c|c|}
\hline $\begin{array}{c}\text { Name } \\
\text { (1) }\end{array}$ & $\begin{array}{l}\text { Type } \\
\text { (2) }\end{array}$ & $\begin{array}{l}\text { Date } \\
\text { (3) }\end{array}$ & $\begin{array}{l}\text { Ref. } \\
\text { star } \\
(4)\end{array}$ & $\begin{array}{c}\text { Comparison } \\
\text { stars } \\
\text { (5) }\end{array}$ & $\begin{array}{c}d o f \\
v_{1}, v_{2} \\
(6)\end{array}$ & $\begin{array}{c}F_{\text {enh }} \\
(7)\end{array}$ & $\begin{array}{c}F_{c}(95 \%) \\
(8)\end{array}$ & $\begin{array}{c}\text { Status } \\
\text { (9) }\end{array}$ & $\begin{array}{l}\text { Amp } \\
(\%) \\
(10)\end{array}$ \\
\hline \multirow[t]{4}{*}{$\mathrm{J} 0324+3410$} & \multirow[t]{4}{*}{ GL } & $24 / 01 / 12$ & $\mathrm{~S} 1$ & $\mathrm{~S} 2, \mathrm{~S} 3$ & 104,208 & 1.39 & 1.31 & V & 12.7 \\
\hline & & $25 / 01 / 12$ & $\mathrm{~S} 1$ & $\mathrm{~S} 2, \mathrm{~S} 3$ & 46,92 & 0.98 & 1.50 & NV & - \\
\hline & & $26 / 01 / 12$ & $\mathrm{~S} 1$ & $\mathrm{~S} 2, \mathrm{~S} 3$ & 88,176 & 3.96 & 1.34 & V & 7.4 \\
\hline & & $02 / 02 / 12$ & $\mathrm{~S} 1$ & $\mathrm{~S} 2, \mathrm{~S} 3$ & 34,68 & 1.05 & 1.60 & NV & - \\
\hline \multirow[t]{3}{*}{$\mathrm{J} 2219+1207$} & \multirow[t]{3}{*}{ GQ } & $29 / 08 / 13$ & $\mathrm{~S} 1$ & $\mathrm{~S} 2, \mathrm{~S} 3$ & 29,58 & 0.63 & 1.66 & $\mathrm{NV}$ & - \\
\hline & & $26 / 08 / 14$ & $\mathrm{~S} 1$ & $\mathrm{~S} 2$ & 26,26 & 3.36 & 1.93 & $\mathrm{~V}$ & 3.1 \\
\hline & & $22 / 09 / 14$ & $\mathrm{~S} 1$ & $\mathrm{~S} 2, \mathrm{~S} 3$ & 72,144 & 1.33 & 1.39 & $\mathrm{NV}$ & - \\
\hline \multirow[t]{2}{*}{ J0351-0526 } & \multirow[t]{2}{*}{ RQ } & $01 / 02 / 14$ & $\mathrm{~S} 2$ & $\mathrm{~S} 1, \mathrm{~S} 3$ & 10,20 & 0.26 & 2.35 & $\mathrm{NV}$ & - \\
\hline & & $02 / 02 / 14$ & $\mathrm{~S} 2$ & $\mathrm{~S} 1, \mathrm{~S} 3$ & 19,38 & 0.47 & 1.87 & $\mathrm{NV}$ & - \\
\hline \multirow{6}{*}{ J0849+5108 } & \multirow[t]{6}{*}{ GL } & $20 / 11 / 12$ & $\mathrm{~S} 2$ & $\mathrm{~S} 1$ & 39,39 & 1.97 & 1.70 & $\mathrm{~V}$ & 12.0 \\
\hline & & $09 / 12 / 12$ & $\mathrm{~S} 2$ & $\mathrm{~S} 1$ & 20,20 & 11.87 & 2.12 & V & 24.4 \\
\hline & & $10 / 12 / 12$ & $\mathrm{~S} 2$ & $\mathrm{~S} 1$ & 19,19 & 0.63 & 2.17 & NV & - \\
\hline & & $25 / 12 / 12$ & $\mathrm{~S} 2$ & $\mathrm{~S} 1$ & 44,44 & 1.28 & 1.65 & NV & - \\
\hline & & $12 / 02 / 13$ & $\mathrm{~S} 2$ & $\mathrm{~S} 1$ & 37,37 & 2.38 & 1.73 & $\mathrm{~V}$ & 36.8 \\
\hline & & $11 / 03 / 13$ & $\mathrm{~S} 2$ & $\mathrm{~S} 1$ & 56,56 & 12.01 & 1.56 & V & 33.3 \\
\hline \multirow{3}{*}{$\mathrm{J} 1613+5247$} & \multirow[t]{3}{*}{ GQ } & $03 / 07 / 13$ & $\mathrm{~S} 1$ & S2 & 12,12 & 8.70 & 2.69 & $\mathrm{~V}$ & 6.6 \\
\hline & & $12 / 07 / 13$ & $\mathrm{~S} 1$ & $\mathrm{~S} 2$ & 24,24 & 1.47 & 1.98 & $\mathrm{NV}$ & - \\
\hline & & $31 / 03 / 14$ & $\mathrm{~S} 2$ & S3 & 25,25 & 2.31 & 1.96 & $\mathrm{~V}$ & 4.4 \\
\hline \multirow[t]{2}{*}{$\mathrm{J} 2123+0102$} & \multirow[t]{2}{*}{ RQ } & $11 / 08 / 12$ & $\mathrm{~S} 2$ & $\mathrm{~S} 1$ & 27,27 & 0.32 & 1.90 & $\mathrm{NV}$ & - \\
\hline & & $03 / 06 / 13$ & $\mathrm{~S} 2$ & $\mathrm{~S} 1, \mathrm{~S} 3$ & 12,24 & 1.31 & 2.18 & NV & - \\
\hline \multirow[t]{4}{*}{ J0948+0022 } & \multirow[t]{4}{*}{ GL } & $26 / 01 / 12$ & $\mathrm{~S} 1$ & S2,S3 & 164,328 & 16.61 & 1.24 & $\mathrm{~V}$ & 51.9 \\
\hline & & $02 / 02 / 12$ & $\mathrm{~S} 1$ & $\mathrm{~S} 2, \mathrm{~S} 3$ & 17,34 & 6.51 & 1.93 & V & 17.1 \\
\hline & & $11 / 03 / 12$ & $\mathrm{~S} 1$ & $\mathrm{~S} 2, \mathrm{~S} 3$ & 39,78 & 45.84 & 1.55 & V & 33.1 \\
\hline & & $19 / 04 / 12$ & $\mathrm{~S} 1$ & $\mathrm{~S} 2, \mathrm{~S} 3$ & 72,144 & 5.8 & 1.39 & V & 25.2 \\
\hline J2339-0912 & GQ & $05 / 10 / 13$ & $\mathrm{~S} 3$ & $\mathrm{~S} 1, \mathrm{~S} 2$ & 23,46 & 0.37 & 1.77 & $\mathrm{NV}$ & - \\
\hline $\mathrm{J} 1326+0334$ & RQ & $30 / 03 / 14$ & $\mathrm{~S} 3$ & $\mathrm{~S} 1, \mathrm{~S} 2$ & 26,52 & 1.38 & 1.71 & $\mathrm{NV}$ & - \\
\hline \multirow[t]{4}{*}{$\mathrm{J} 1505+0326$} & \multirow[t]{4}{*}{ GL } & $18 / 04 / 12$ & $\mathrm{~S} 1$ & $\mathrm{~S} 2, \mathrm{~S} 3$ & 23,46 & 0.65 & 1.77 & NV & - \\
\hline & & $22 / 05 / 12$ & $\mathrm{~S} 1$ & $\mathrm{~S} 2, \mathrm{~S} 3$ & 10,20 & 1.10 & 2.35 & NV & - \\
\hline & & $23 / 05 / 12$ & $\mathrm{~S} 1$ & $\mathrm{~S} 2, \mathrm{~S} 3$ & 12,24 & 0.51 & 2.18 & NV & - \\
\hline & & $24 / 05 / 12$ & $\mathrm{~S} 1$ & $\mathrm{~S} 2, \mathrm{~S} 3$ & 11,22 & 5.44 & 2.26 & $\mathrm{~V}$ & 10.1 \\
\hline \multirow[t]{5}{*}{$\mathrm{J} 1256+3852$} & \multirow[t]{5}{*}{ GQ } & $10 / 04 / 13$ & $\mathrm{~S} 2$ & S3 & 22,22 & 1.61 & 2.05 & $\mathrm{NV}$ & - \\
\hline & & $05 / 01 / 14$ & $\mathrm{~S} 2$ & S3 & 9,9 & 3.91 & 3.18 & V & 7.1 \\
\hline & & $01 / 02 / 14$ & $\mathrm{~S} 2$ & S3 & 8,8 & 4.49 & 3.44 & V & 4.8 \\
\hline & & $02 / 02 / 14$ & $\mathrm{~S} 2$ & $\mathrm{~S} 1, \mathrm{~S} 3$ & 8,16 & 0.54 & 2.59 & $\mathrm{NV}$ & - \\
\hline & & $01 / 05 / 14$ & $\mathrm{~S} 2$ & $\mathrm{~S} 1, \mathrm{~S} 3$ & 19,38 & 0.58 & 1.87 & $\mathrm{NV}$ & - \\
\hline \multirow[t]{2}{*}{ J0037-0933 } & \multirow[t]{2}{*}{ RQ } & $16 / 10 / 12$ & $\mathrm{~S} 2$ & $\mathrm{~S} 1, \mathrm{~S} 3$ & 11,22 & 1.86 & 2.26 & $\mathrm{NV}$ & - \\
\hline & & $06 / 10 / 13$ & $\mathrm{~S} 2$ & $\mathrm{~S} 1, \mathrm{~S} 3$ & 20,40 & 0.60 & 1.84 & NV & - \\
\hline
\end{tabular}

tour of the source from FIRST survey generated using AIPS ${ }^{5}$ is shown in Fig. 1. It has a radio loudness parameter $(\log \mathrm{R})$ smaller than 1 which is the dividing value between radio-loud and radioquiet AGN as proposed by Kellermann et al. (1989). This low value might be attributed to an overestimate of the optical flux due the underlying host galaxy which is clearly visible in the optical images.

\footnotetext{
5 AIPS is produced and maintained by the National Radio Astronomy Observatory, a facility of the National Science Foundation operated under cooperative agreement by Associated Universities, Inc.
}

This source has been observed for three nights over a period of 1 year for durations ranging between about 3 to 7.6 hours. For statistical calculations, S1 is taken as the reference star and S2, S3 are taken as the comparison stars on all the nights except $26 \mathrm{Au}-$ gust 2014 on which only S2 is used as the comparison star as S3 varies (Fig. 2). Seeing is found to be steady on all the three nights of observations. On the first night, the object is found to be nonvariable (NV). However it shows unambiguous INOV of the order of 0.03 magnitudes on the second night. On the third night, when it was monitored for the longest duration, the densely sampled light 

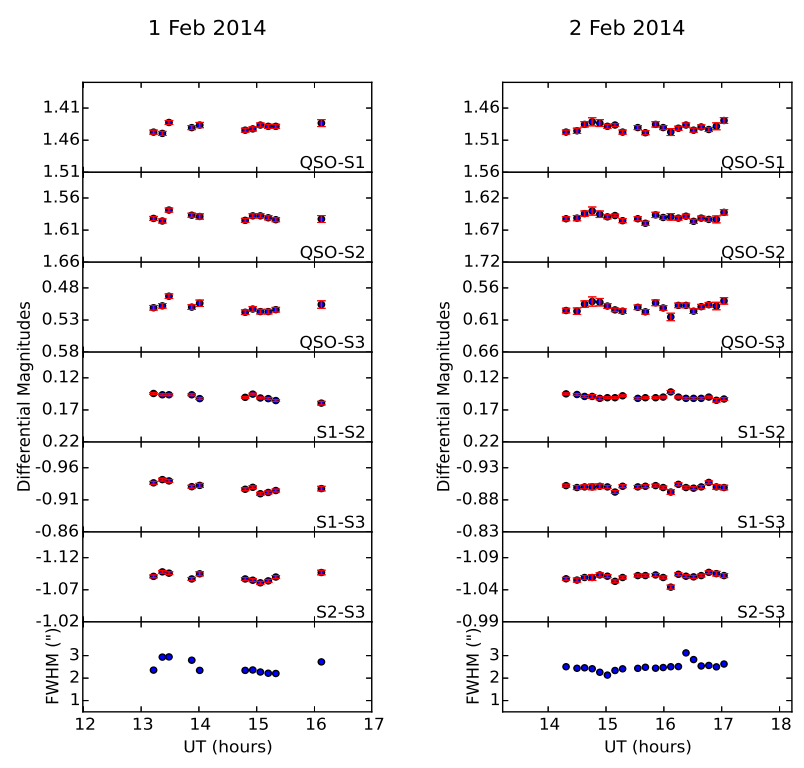

Figure 3. DLCs of the RQ-NLSy1 galaxy, J0351-0526.

curves with 73 epochs of observation over 7.6 hours shows no INOV. In the long term, it has shown both brightening and dimming behaviour during our observations. Between 29 August 2013 and 26 August 2014 the source has brightened by 0.2 mag and faded by the same amount when observed a month later on 29 September 2016.

Set 1 - J0351-0526: This RQ-NLSy1 galaxy was monitored for two consecutive days in February 2014 for about 3 hours on both the nights. For statistical analysis of variability, the reference star used is S2. S1 and S3 are used as the comparison stars (Fig. 3). It is classified as non-variable.

Set 2 - J1613+5247: This radio-loud NLSy1 galaxy with log $\mathrm{R}=1.04$ was observed for three nights over a period of eight months between July 2013 and March 2014. The DLC of stars S1 and S2 has the lowest standard deviation of 0.0076 on two nights and thus has been considered as the steadiest pair of stars on those two nights for statistical analysis of variability of which $S 1$ is taken as the reference star (Fig. 4). However, on one night (31 March 2014) stars S2 and S3 are taken as the reference star and comparison star respectively, as the star $\mathrm{S} 1$ is found to be unsteady. This source has shown definite INOV on 03 July 2013 with INOV amplitude of $6.6 \%$ and on 31 March 2014 with amplitude 4.4\%. On 12 July 2013 it is non-variable.

Set 2 - J2123+0102: This radio-quiet source was monitored for two nights. The reference star used for the statistical analysis is S2. Stars S1, S3 are used for comparison except on 11 August 2012 where only S1 is used as S3 varies (Fig. 5). The source is non-variable on both the nights. Long term optical variability is seen in this source, wherein, it faded by about 0.2 magnitudes over a 10 month period between 11 August 2012 and 3 June 2013.

Set 3 - J2339-0912: This radio-loud NLSy1 galaxy has the highest radio flux density of $4.39 \mathrm{mJy}$ at $1.4 \mathrm{GHz}$ with a radio-loudness parameter $(\log \mathrm{R})$ of 1.79 , close to the dividing line between radio-loud and very radio-loud NLSy1 galaxies (Kellermann et al. 1989). It has a steep radio spectral index $\alpha=$ -1.4 between 6 and 20cm (Glikman et al. 2007) and the NVSS contour map points to an extended structure. It did not show any unambiguous evidence of INOV on the single night it was observed
Table 5. Duty cycles, mean amplitude of variability and the corresponding error in amplitude (in\%), for different classes of NLSy1 galaxies

\begin{tabular}{llllll}
\hline & GL & GQ & $\begin{array}{l}\text { RQ } \\
(3)\end{array}$ & $\begin{array}{l}\text { HP } \\
(5)\end{array}$ & $\begin{array}{l}\text { LP } \\
(6)\end{array}$ \\
\hline DC & 55.2 & 38.8 & 0.0 & 69.0 & 58.9 \\
\hline Mean Amp & 24.0 & 5.2 & 0.0 & 29.2 & 10.0 \\
& \pm 13.7 & \pm 1.6 & \pm 0.0 & \pm 12.4 & \pm 3.8 \\
\hline
\end{tabular}

(Fig. 6). For statistical tests, the comparison stars used are S1 and $\mathrm{S} 2$, whereas $\mathrm{S} 3$ is taken as the reference star.

Set 3 - J1326+0334: This RQ-NLSy1 was observed for only one epoch. Based on the statistical test, we classify this source as $\mathrm{NV}$ on that night (Fig. 7). The reference star used is S3 and S1, S2 are taken as the comparison stars.

Set 4 - J1256+3852: This radio-loud source was monitored for five epochs between April 2013 and May 2014. The steadiest pair of stars are S2 and S3 (standard deviation $~ 0.0053$ ) of which $\mathrm{S} 2$ is taken as the reference star. S1 is found to vary on the first three nights and hence is not used in the analysis for those nights. However, on the remaining two nights (2 February 2014 and 1 May 2014) it is included as the second comparison star. This NLSy1 galaxy shows clear evidence of INOV on two nights, 5 January 2014 and 1 February 2014, with amplitude of variability of $7.1 \%$ and $4.8 \%$ respectively (Fig. 8). Over a period of five months between 5 January and 01 May, 2014, the object has steadily brightened by about $0.075 \mathrm{mag}$.

Set 4 - J0037-0933: Classified as radio-quiet, this source was observed for two epochs, separated by a year. On both the nights it is NV. For characterizing the variability, the reference star used is S2 and stars S1, S3 are taken as the comparison stars (Fig. 9).

\section{RESULTS AND DISCUSSIONS}

Our results of INOV show clear contrast between RQ and RLNLSy1 galaxies that includes GL-NLSy1 and GQ-NLSy1 galaxies. None of the RQ-NLSy1 galaxies show INOV whereas three out of the four GQ-NLSy1 galaxies show INOV. When compared with the INOV characteristics of GL-NLSy1 galaxies, all the four GL-NLSy1 galaxies show INOV, the results of which are already available in literature. The DC of INOV is the highest in the case of GL-NLSy1 galaxies with a value of about $55 \%$. This is followed by GQ-NLSy1 galaxies with a DC of about $39 \%$. Thus, the observations reported here find clear differences between the duty cycles of INOV in different classes of NLSy1 galaxies. Also, GLNLSy1 galaxies show large amplitude variations compared to the other two classes. The amplitude of INOV shown by GL-NLSy1 galaxies range from $7 \%$ to $52 \%$ with a mean of $24.0 \pm 13.7 \%$. In contrast, GQ-NLSy1 galaxies show milder INOV ranging from $3 \%$ to $7 \%$ with an average of $5.2 \pm 1.6 \%$. The non-detection of INOV in the limited sample RQ-NLSy1 galaxies studied here, need not necessarily imply the absence of INOV in this class of NLSy1 galaxies. A larger sample with more monitoring observations could result in INOV detection.

The results obtained for NLSy1 galaxies in this work are in close agreement with that known for quasars, with blazars showing large amplitude and high DC of variability than radio-quiet quasars that show low DC. The contrasting INOV behaviour be- 
3 Jul 2013

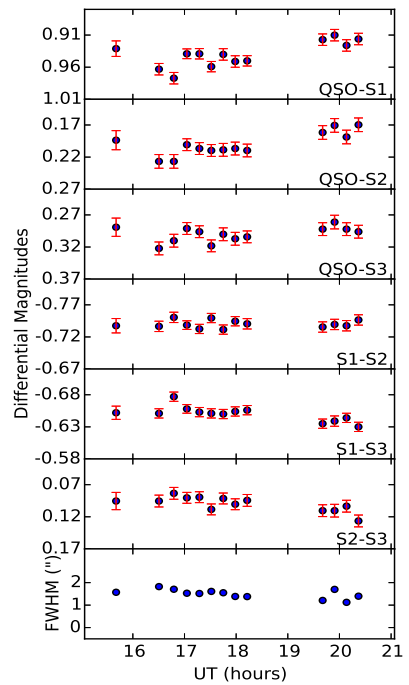

12 Jul 2013

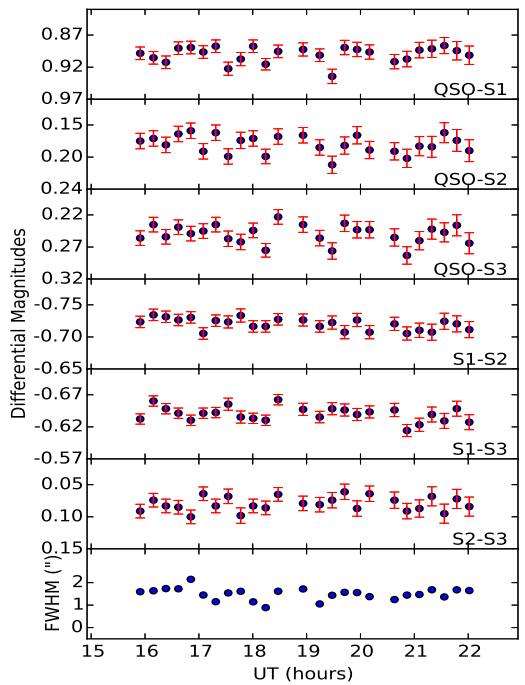

31 Mar 2014

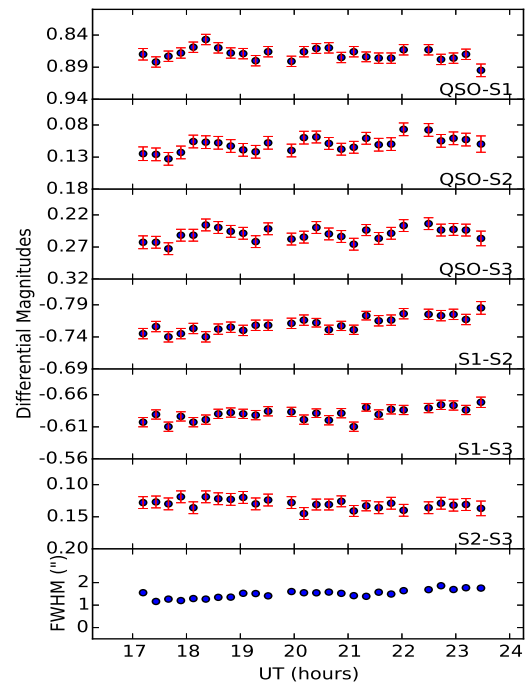

Figure 4. DLCs of the GQ-NLSy1 galaxy, J1613+5247.

11 Aug 2012

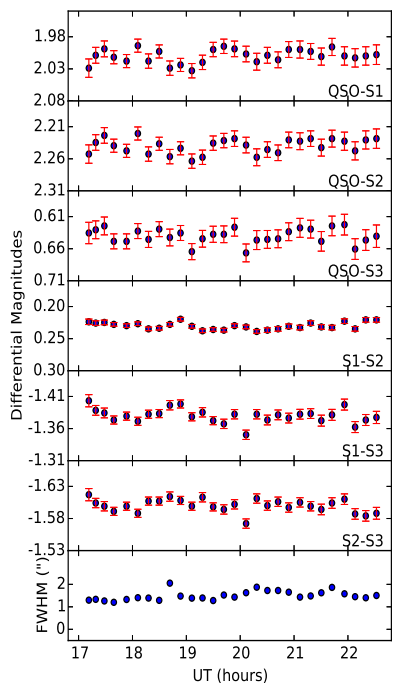

3 June 2013

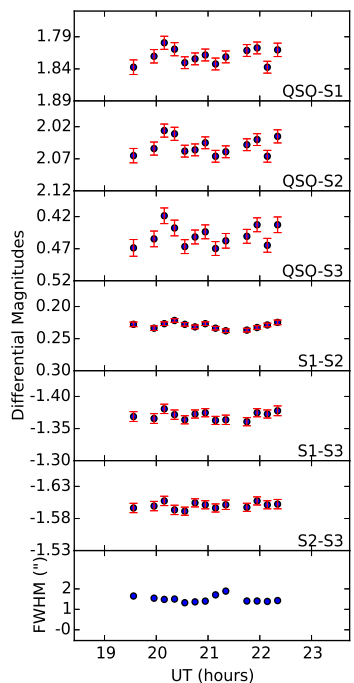

Figure 5. DLCs of the RQ-NLSy1 galaxy, J2123+0102.

tween RQ and RL-NLSy1 galaxies as well as between RQ and RL-quasars can naturally be explained by relativistic beaming arguments (Gopal-Krishna et al. 2003). However, optical polarization observations in the recent past on FSRQs, separated in two groups namely low polarization FSRQs and high polarization FSRQs, clearly show that high polarization FSRQs are more variable (high DC and amplitude) than their less polarized counterparts. This suggest that mere presence of relativistically beamed jet is not sufficient to explain high INOV. Alternatively, the close linkage between optical polarization and INOV (Goyal et al. 2012) indicates that optical polarization is the crucial factor for causing INOV. This is quite on expected lines as optical polarization is closely connected to shocks in relativistically beamed jets in blazars.
Three of the four GL-NLSy1 galaxies considered in this work, have optical polarization observations. $1 \mathrm{H} 0323+343$ has polarization value around $1 \%$. However it does show a flaring state with polarization value close to $3 \%$ (Itoh et al. 2014). For SBS $0846+513$ and PMN J0948+0022, polarization as high as $10 \%$ (Maune et al. 2014) and 36\% (Itoh et al. 2013) have been known. Optical polarization measurements are not known for other sources in our sample. Dividing the GL-NLSy1 galaxies into high polarization (HP) (with P > 3\%) and low polarization (LP) NLSy1 galaxies, we find a DC cycle of about $69 \%$ and $59 \%$ for HP and LPNLSy1 galaxies respectively. This is in close analogy with what is seen in blazars. (Goyal et al. 2012). Also, GL-blazars are found to show more polarization in the optical band than GQ-blazars 
30 Mar 2014

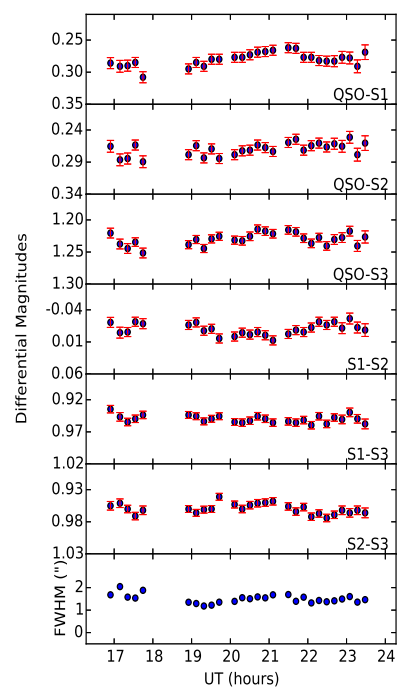

Figure 7. DLC of the RQ-NLSy1 galaxy, J1326+0334.

(Angelakis et al. 2016). In the population of GL-NLSy1 galaxies, sources with strong optical polarization show large amplitude INOV $(29.2 \pm 12.4 \%)$ compared to their less polarized counterparts $(10.0 \pm 3.8 \%)$. However, the caveat here is that the polarization data used here (that are compiled from literature) has not been acquired simultaneous to our INOV observation. In spite of this short coming, results found here clearly suggest that high polarization GLNLSy1 galaxies are more variable within a night than their counterparts with low optical polarization. To firmly establish this point, simultaneous optical flux and polarization observations on a large number of sources are needed. This is not feasible at present as there are only about half a dozen of $\gamma$-ray emitting NLSy1 galaxies known. However, it is expected to be achievable in the future when more number of $\gamma$-ray emitting NLSy1 galaxies would be detected.

In this work we want to quantify the INOV characteristics of the different categories of NLSy1 galaxies to understand the similarities and differences among them as well as to compare with that of their luminous counterparts, the quasars. This is motivated by the detection of $\gamma$-ray emission from few RL-NLSy1 galaxies by Fermi. Available studies do indicate that RL-AGN are hosted by black holes having high masses in excess of $10^{8} M_{\odot}$ (Laor 2000; Chiaberge \& Marconi 2011). The detection of $\gamma$-ray emission in RL-NLSy1 galaxies believed to have BH masses of the order of $10^{6}-10^{7} M_{\odot}$ (Xu et al. 2012) is a direct challenge to this idea. Irrespective of this, GL-NLSy1 galaxies have now emerged as a new class of $\gamma$-ray emitting AGN, though the number of objects known as of now are limited. This is likely due the small number of NLSy1 galaxies known till date (Zhou et al. 2006). A multitude of observations on most of the GL-NLSy1 galaxies known as of now, do indicate that most of the properties of these sources are analogous to blazars, such as large INOV (Liu et al. 2010; Paliya et al. 2013a, 2016) broad band SED (Paliya et al. 2016, 2014) compact core-jet structure in the radio (D'Ammando et al. 2012; Orienti et al. 2012; D'Ammando et al. 2013a, 2014), superluminal motion (D'Ammando et al. 2013b), optical polarization (Itoh et al. 2014, 2013; Maune et al. 2014) etc. All these properties do indicate the presence of relativistic jets in these sources, similar to blazars. As the broad band SED and gamma-ray spec- tral properties of these sources have close resemblance to FSRQs, it has been postulated that they are the low mass black hole counterparts to FSRQs (Paliya et al. 2016). However, this assertion is unlikely to hold long if it could be conclusively established that these sources do have BH masses like blazars. Recent reports do indicate little differences between the black hole masses of GLNLSy1 galaxies and blazars. Examples are the determination of $\mathrm{BH}$ mass of $6 \times 10^{8} M_{\odot}$ in the GL-NLSy1 galaxy PKS 2004-0447 from spectro-polarimetry (Baldi et al. 2016) using the broad $H_{\alpha}$ emission line $(\mathrm{FWHM}=9000 \mathrm{~km} / \mathrm{sec})$ in polarized light, and masses of $1.6 \times 10^{9} M_{\odot}$ and $3.2 \times 10^{8} M_{\odot}$ respectively in PMN J0948+0022 and PKS 1502+036 via accretion disk modeling (Calderone et al. 2013). Thus, recent observations including the one presented here point to close similarity between GL-NLSy1 galaxies and blazars. However, ambiguity still remains on the host galaxy of GL-NLSy1 galaxies (spirals v/s ellipticals) compared to blazars that are hosted by ellipticals. (León Tavares et al. 2014; Kotilainen et al. 2016)

\section{SUMMARY}

We have reported results of a first comparative study on the INOV behaviour of three different classes of NLSy1 galaxies, namely, RQ, GQ and GL-NLSy1 galaxies. Though the observations of RQ and GQ-NLSy1 galaxies are reported for the first time, INOV studies of GL-NLSy1 galaxies were available in the literature (Liu et al. 2010; Paliya et al. 2013a, 2016). Taking the observations of GLNLSy1 galaxies reported by us earlier, the mean observing time for the sample of GL-NLSy1 galaxies is 3.8 hours over 18 nights. For RQ-NLSy1 and GQ-NLSy1 galaxies sample the mean monitoring time are 4.6 hours over 7 nights and 4.4 hours over 12 nights respectively. Considering the error in the photometry $(\sigma=0.0054)$, our observations are sensitive to detect INOV above the $1 \%$ level corresponding to $95 \%$ confidence $(2 \sigma)$, which forms the detection threshold of our observations reported here. The power-enhanced F-test was adopted to detect INOV. The findings of this study are:

(i) INOV has been detected in RL-NLSy1 galaxies but not in RQ-NLSy1 galaxies.

(ii) RL-NLSy1 galaxies that include both GL and GQ-NLSy1 galaxies show large DC of INOV of 50\% in sharp contrast to the null DC shown by RQ-NLSy1 galaxies. Thus there is a marked difference in the INOV properties between RL and RQ-NLSy1 galaxies. This is also in close agreement with what is known of the INOV properties of the luminous counterparts to NLSy1 galaxies, i.e. RLquasars show INOV more frequently than RQ-quasars.

(iii) Among RL-NLSy1 galaxies, GL-NLSy1 show the highest DC of variability of about $55.2 \%$ using the adopted variability statistics. Their amplitude of variability is also larger than the other classes of NLSy1 galaxies. They show a large mean variability amplitude of $24.0 \pm 13.7 \%$ compared to GQ-NLSy1 galaxies $(5.2 \pm$ $1.6 \%$ ). Such extreme INOV behaviour shown by GL-NLSy1 galaxies might be due to the high bulk Lorentz factor of their inner jets. The null DC of INOV shown by RQ-NLSy1 galaxies could be due to a less powerful or a misaligned (and consequently a lower $\delta$ ) jet compared to the other classes of NLSy1 galaxies.

(iv) Dividing the GL-NLSy1 galaxies into HP-NLSy1 and LPNLSy1 galaxies based on their optical polarization properties, we find that HP-NLSy1 galaxies show DC (amplitude) of variations of about 69.0(29.2 \pm 12.4$) \%$, which appear to be somewhat higher than the values of $58.9(10.0 \pm 3.8) \%$ shown by the LP-NLSy1 galaxies. 

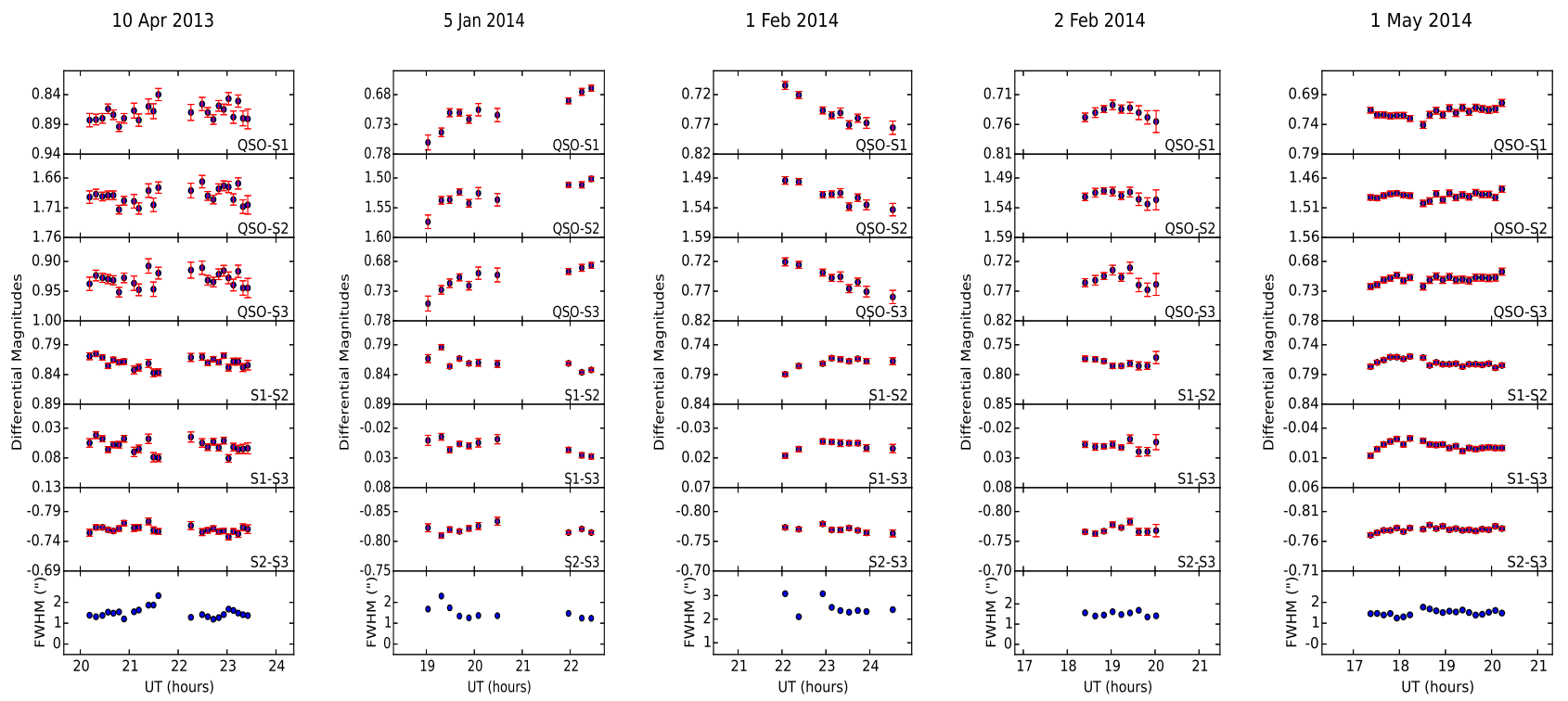

Figure 8. DLCs of the GQ-NLSy1 galaxy, J1256+3852.

16 Oct 2012

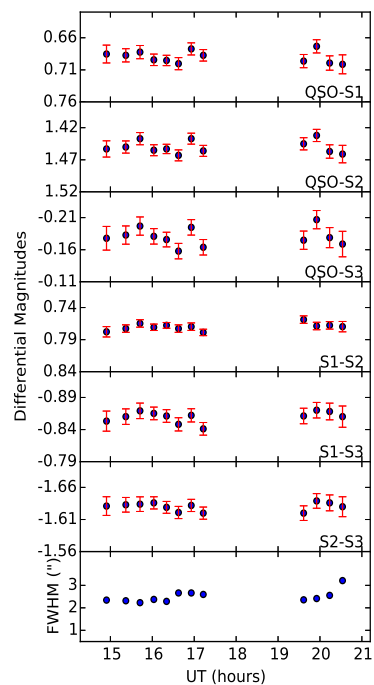

6 Oct 2013

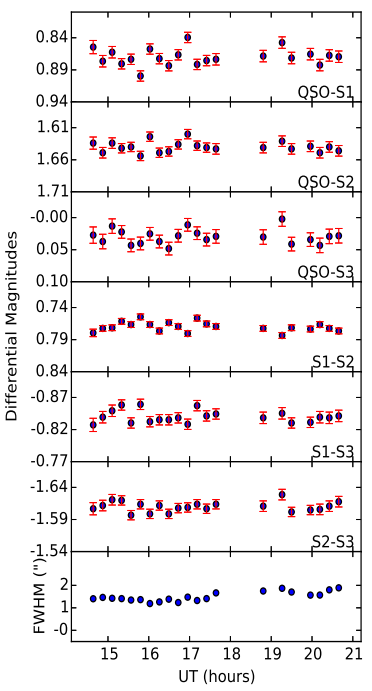

Figure 9. DLCs of the RQ-NLSy1 galaxy, J0037-0933.

\section{ACKNOWLEDGEMENTS}

We thank the referee Prof. Paul Wiita for his critical comments on the manuscript. We also thank the staff of IAO, Hanle and CREST, Hoskote, who made these observations possible. The facilities at IAO and CREST are operated by the Indian Institute of Astrophysics, Bangalore.

\section{REFERENCES}

Abdo A. A., et al., 2009a, ApJ, 699, 976

Abdo A. A., et al., 2009b, ApJ, 707, L142

Abdo A. A., et al., 2010, ApJ, 722, 520

Ackermann M., et al., 2016, ApJ, 824, L20

Angelakis E., et al., 2016, MNRAS,

Baldi R. D., Capetti A., Robinson A., Laor A., Behar E., 2016, MNRAS, 458, L69

Blundell K. M., Rawlings S., 2001, ApJ, 562, L5

Boroson T. A., 2002, ApJ, 565, 78

Calderone G., Ghisellini G., Colpi M., Dotti M., 2013, MNRAS, 431, 210

Carini M. T., 1990, PhD thesis, , George State University, Atlanta, USA, (1990)

Carini M. T., Miller H. R., Noble J. C., Sadun A. C., 1991, AJ, 101, 1196

Cellone S. A., Romero G. E., Combi J. A., 2000, AJ, 119, 1534

Chiaberge M., Marconi A., 2011, MNRAS, 416, 917

D’Ammando F., et al., 2012, MNRAS, 426, 317

D'Ammando F., et al., 2013a, MNRAS, 433, 952

D'Ammando F., et al., 2013b, MNRAS, 436, 191

D'Ammando F., et al., 2014, MNRAS, 438, 3521

D'Ammando F., Orienti M., Larsson J., Giroletti M., 2015, MNRAS, 452,520

Foschini L., Colpi M., Gallo L., Grupe D., Komossa S., Leighly K., Mathur S., eds, 2011, Narrow-Line Seyfert 1 Galaxies and their place in the Universe

Foschini L., et al., 2015, A\&A, 575, A13

Gaur H., et al., 2015, MNRAS, 452, 4263

George I. M., Turner T. J., Yaqoob T., Netzer H., Laor A., Mushotzky R. F., Nandra K., Takahashi T., 2000, ApJ, 531, 52

Glikman E., Helfand D. J., White R. L., Becker R. H., Gregg M. D., Lacy M., 2007, ApJ, 667, 673

Gopal-Krishna Stalin C. S., Sagar R., Wiita P. J., 2003, ApJ, 586, L25

Gopal-Krishna Joshi R., Chand H., 2013, MNRAS, 430, 1302

Goyal A., Gopal-Krishna Wiita P. J., Anupama G. C., Sahu D. K., Sagar R., Joshi S., 2012, A\&A, 544, A37

Goyal A., Mhaskey M., Gopal-Krishna Wiita P. J., Stalin C. S., Sagar R., 2013, Journal of Astrophysics and Astronomy, 34, 273

Heidt J., Wagner S. J., 1996, A\&A, 305, 42

Howell S. B., 1989, PASP, 101, 616

Hughes P. A., Aller H. D., Aller M. F., 1992, ApJ, 396, 469

Itoh R., et al., 2013, ApJ, 775, L26

Itoh R., et al., 2014, PASJ, 66, 108

Ivezić Ž., et al., 2002, AJ, 124, 2364

Jiang N., et al., 2012, ApJ, 759, L31

Joshi R., Chand H., Gupta A. C., Wiita P. J., 2011, MNRAS, 412, 2717

Kellermann K. I., Sramek R., Schmidt M., Shaffer D. B., Green R., 1989, 
AJ, 98, 1195

Klimek E. S., Gaskell C. M., Hedrick C. H., 2004, ApJ, 609, 69

Komossa S., Voges W., Xu D., Mathur S., Adorf H.-M., Lemson G., Duschl W. J., Grupe D., 2006, AJ, 132, 531

Kotilainen J. K., Tavares J. L., Olguin-Iglesias A., Baes M., Anorve C., Chavushyan V., Carrasco L., 2016, preprint, (arXiv: 1609.02417)

Laor A., 2000, ApJ, 543, L111

León Tavares J., et al., 2014, ApJ, 795, 58

Liu H., Wang J., Mao Y., Wei J., 2010, ApJ, 715, L113

Marchesini E. J., et al., 2016, preprint, (arXiv: 1608.05429)

Marscher A. P., Gear W. K., 1985, ApJ, 298, 114

Marscher A. P., Gear W. K., Travis J. P., 1992, in Valtaoja E., Valtonen M., eds, Variability of Blazars. p. 85

Mattox J. R., et al., 1996, ApJ, 461, 396

Maune J. D., Miller H. R., Eggen J. R., 2011, in Narrow-Line Seyfert 1 Galaxies and their Place in the Universe. p. 59

Maune J. D., Eggen J. R., Miller H. R., Marshall K., Readhead A. C. S., Hovatta T., King O., 2014, ApJ, 794, 93

Miller H. R., Carini M. T., Goodrich B. D., 1989, Nature, 337, 627

Miller P., Rawlings S., Saunders R., 1993, MNRAS, 263, 425

Miller H. R., Ferrara E. C., McFarland J. P., Wilson J. W., Daya A. B., Fried R. E., 2000, New Astron. Rev., 44, 539

Oke J. B., 1974, ApJS, 27, 21

Orienti M., D'Ammando F., Giroletti M., for the Fermi-LAT Collaboration 2012, preprint, (arXiv: 1205.0402)

Osterbrock D. E., Pogge R. W., 1985, ApJ, 297, 166

Paliya V. S., Stalin C. S., Kumar B., Kumar B., Bhatt V. K., Pandey S. B., Yadav R. K. S., 2013a, MNRAS, 428, 2450

Paliya V. S., Stalin C. S., Shukla A., Sahayanathan S., 2013b, ApJ, 768, 52

Paliya V. S., Sahayanathan S., Parker M. L., Fabian A. C., Stalin C. S., Anjum A., Pandey S. B., 2014, ApJ, 789, 143

Paliya V. S., Böttcher M., Diltz C., Stalin C. S., Sahayanathan S., Ravikumar C. D., 2015, ApJ, 811, 143

Paliya V. S., Rajput B., Stalin C. S., Pandey S. B., 2016, ApJ, 819, 121

Polednikova J., et al., 2016, MNRAS, 460, 3950

Rodríguez-Ardila A., Binette L., Pastoriza M. G., Donzelli C. J., 2000, ApJ, 538,581

Romero G. E., Cellone S. A., Combi J. A., 1999, A\&AS, 135, 477

Sagar R., Stalin C. S., Gopal-Krishna Wiita P. J., 2004, MNRAS, 348, 176

Stalin C. S., Gopal Krishna Sagar R., Wiita P. J., 2004a, Journal of Astrophysics and Astronomy, 25, 1

Stalin C. S., Gopal-Krishna Sagar R., Wiita P. J., 2004b, MNRAS, 350, 175

Urry C. M., Padovani P., 1995, PASP, 107, 803

Véron-Cetty M.-P., Véron P., 2010, A\&A, 518, A10

Wagner S. J., Witzel A., 1995, ARA\&A, 33, 163

Xu D., Komossa S., Zhou H., Lu H., Li C., Grupe D., Wang J., Yuan W., 2012, AJ, 143, 83

Yao S., Yuan W., Zhou H., Komossa S., Zhang J., Qiao E., Liu B., 2015, MNRAS, 454, L16

Zhou H., Wang T., Yuan W., Lu H., Dong X., Wang J., Lu Y., 2006, ApJS, 166,128

de Diego J. A., 2014, AJ, 148, 93

de Diego J. A., Polednikova J., Bongiovanni A., Pérez García A. M., De Leo M. A., Verdugo T., Cepa J., 2015, AJ, 150, 44

This paper has been typeset from a $\mathrm{T}_{\mathrm{E}} \mathrm{X} / \mathrm{L} \mathrm{T}_{\mathrm{E}} \mathrm{X}$ file prepared by the author. 